\title{
La mujer y el liderazgo empresarial*
}

\section{Women and Business Leadership}

\author{
Françoise Contreras Torres** \\ Universidad del Rosario, Bogotá-Colombia \\ Julián Eduardo Pedraza Ortiz \\ Universidad del Rosario, Bogotá-Colombia \\ Ximena Mejía Restrepo \\ Universidad del Rosario, Bogotá-Colombia \\ Recibido: 4 de septiembre de 2011 \\ Revisado: 4 de octubre de 2011 \\ Aceptado: 17 de diciembre de 2011
}

\section{Resumen}

El objetivo de este estudio fue describir la situación actual de la mujer en el mundo laboral y analizar las circunstancias que le han impedido alcanzar altas posiciones en las compañías. El núcleo de la discusión giró en torno a las barreras del liderazgo femenino y cómo estas pueden ser superadas a través del reconocimiento de las diferencias. Se argumenta que el principal problema relacionado con la participación inequitativa en cargos de alta gerencia se debe principalmente a los estereotipos femeninos, los cuales suelen ser contrarios a los roles de liderazgo aceptados por la mayoría de las culturas. Se resalta la necesidad de que las mujeres ejerzan el liderazgo desde sus propias características, en vez de imitar el tradicional rol de liderazgo masculino.

Palabras clave: liderazgo en las mujeres, estereotipos femeninos, género, equidad laboral, alta gerencia femenina.

Artículo de investigación. Línea de investigación en Liderazgo del Grupo de Investigación en Perdurabilidad Empresarial (GIPE), Universidad del Rosario.

** Correspondencia: Françoise Contreras, Facultad de Administración, Universidad del Rosario, Bogotá, Colombia. Correo electrónico: francoise. contreras@urosario.edu.co, correo electrónico: pedraza.julian@ur.edu.co, correo electrónico: mejia.ximena@ur.edu.co Dirección postal: cra 5 No. 15-37 of. 201. Universidad del Rosario. 


\section{Abstract}

The aim of this paper was to describe the current leadership situation from the gender perspective and to analyze the circumstances that deter women from achieving high leadership positions in companies. The core of the discussion turned around women's leadership barriers and how these can be overcome through the recognition of the difference of being a woman. We argued that the main issue related to this unequal participation as CEOs is mainly due to stereotypes concerning women's roles, which in many cases are contrary to the leadership roles accepted in most cultures. We point out that women should find their own leadership capacity, according to their own characteristics rather than to imitate the traditional male leadership role.

Keywords: women's leadership, female stereotypes, gender, work's equity, female top executives.

En las últimas décadas, el ingreso de las mujeres al mundo laboral ha tenido un crecimiento acelerado; sin embargo, su ascenso en la escala corporativa ha mostrado ser más lento. De acuerdo con los datos existentes a nivel global, la presidencia de las compañías y la alta gerencia sigue estando a cargo principalmente de varones, situación evidentemente inequitativa, si se consideran las actuales condiciones de las mujeres a nivel educativo y social. Este fenómeno aún no es suficientemente claro y la explicación desde las capacidades vinculadas al sexo se agota a sí misma, el asunto parece estar más relacionado con variables psicológicas, sociales y culturales que deben ser estudiadas a profundidad desde diversos métodos de análisis para alcanzar una mayor comprensión de la situación e intervenir favorablemente para promover la equidad.

El propósito de este estudio es abordar esta problemática a partir de una revisión documental que dé cuenta de la situación actual de la mujer en el mundo laboral y analizar las circunstancias que le han impedido ocupar altos cargos en las empresas, en proporciones similares a las de los hombres.

Para lograr tal objetivo, en esta revisión teórica se expondrán algunos datos respecto a la ocupación actual de la mujer en altos cargos directivos, sus condiciones a nivel laboral, social y educativo, y se comentará su inminente, aunque lento, ascenso a la alta dirección. Posteriormente, se discutirá en torno a las barreras que sustentan la inequidad para ocupar estos cargos y se resaltará la necesidad de superar la búsqueda de la igualdad, pues justamente el reconocer las diferencias biológicas, sociales, psicológicas y culturales entre hombres y mujeres permitirá alcanzar la equidad, favoreciendo así el desarrollo de las mujeres como líderes empresariales.

\section{Situación actual y procesos de cambio}

Aunque la participación de las mujeres en altos cargos directivos y gerenciales se ha incrementado en las últimas décadas, su participación en la cima de la escala organizacional sigue siendo muy reducida; un ejemplo de ello es que de las 500 empresas más grandes del mundo, solo el $1 \%$ son presididas por mujeres. Lo anterior guarda relación con los salarios, pues de los ejecutivos mejor pagados en Estados Unidos apenas el 6\% son mujeres y el $2 \%$ llegan a ser presidentes ejecutivas. En algunos países de la Unión Europea la situación mejora un poco, pero el fenómeno es similar; los altos cargos gerenciales son ocupados por mujeres en un $11 \%$ y llegan a presidir las empresas solo un 4\% (Eagly \& Carli, 2007). Como habría de esperar, la participación de las mujeres en las juntas de accionistas de las empresas es también significativamente menor; de acuerdo con los datos, en el año 2007 en Estados Unidos estas juntas contaban con un $14,8 \%$ de mujeres, porcentaje similar en Canadá $(13,7 \%)$ y más alto que en Francia y España, países en los 
que la participación femenina no supera el $8 \%$ (Henrekson \& Stenkula, 2009).

En Colombia tampoco hay participación paritaria en altos cargos de liderazgo; no obstante, el índice de mujeres que lo alcanzan es mayor que en otros países latinoamericanos, doblando en proporción a México y Venezuela (Maxfield, 2007). Dicha variación entre países obedece a varias razones, entre ellas, el tipo de actividad empresarial, el nivel de desarrollo, las características culturales y el nivel de acceso a la educación, entre otros; pero, en cualquier caso, la participación femenina en estos cargos sigue siendo notablemente menor (Guzmán \& Rodríguez, 2008).

A pesar de la situación descrita, es innegable el importante posicionamiento laboral que han alcanzado las mujeres actualmente y la perspectiva que se tiene sobre su inminente ascenso en la pirámide corporativa en un futuro cercano. Dicho avance ha sido producto del esfuerzo de las mujeres por la igualdad de oportunidades, el cual se hizo más evidente en la mitad del siglo pasado, cuando la presión por parte de distintos estamentos sociales por lograr un trato más equitativo permitió el avance hacia la construcción de nuevas formas de pensamiento (Rodríguez de Lecea, 2008). Cabe resaltar que estos movimientos sociales no hacen parte de la historia reciente; se iniciaron en el siglo XVIII con la revolución francesa, movimiento social en el que se resaltaron los ideales de igualdad y de justicia, promoviendo una sociedad incluyente en la que se reconociera el rol social de hombres y mujeres, condición que sustentaba el lema de la revolución: Libertad, Igualdad y Fraternidad, ideales que hoy, después de tantos años, no se han cumplido (Sánchez, 1991).

A nivel laboral, las condiciones de inequidad se manifiestan en menores ingresos para la mujer y menos oportunidades para ascender en la jerarquía organizacional, a pesar de su nivel de formación profesional (Godoy \& Mladinic, 2009). La inequidad salarial en razón del sexo sucede en casi todas las naciones del mundo, en las que los hombres ganan más dinero que las mujeres ocupando el mismo cargo, con un nivel de forma- ción similar y contando con las mismas habilidades (Fernández, 2006). De acuerdo con la CEPAL (2010), el promedio mundial de la diferencia salarial entre hombres y mujeres está en el $66 \%$ con algunas variaciones dependiendo del nivel educativo alcanzado: con un alto nivel, las mujeres alcanzan el $69 \%$ del salario de un hombre, un $76 \%$ con nivel medio y un $68 \%$ cuando el nivel de formación es escaso. Es así que aunque se haya alcanzado la paridad en materia educativa en el 2005, esto no se ha traducido en un aumento equivalente en los ingresos ni en la calidad del empleo al que ellas pueden acceder.

En este sentido, aunque la educación ha sido uno de los grandes logros sociales, no ha sido un factor determinante para la ubicación laboral de las mujeres, pues esta sigue obedeciendo a parámetros tradicionales que no les permite acceder a trabajos acordes con su nivel de formación (Gálvez, 2001). El hecho de que las mujeres logren desempeñarse en posiciones de liderazgo, representa su interés por romper el llamado techo de cristal que les dificulta la posibilidad de acceder a altos cargos directivos y les brinda la oportunidad de ir cambiando estos patrones sociales y culturales que fomentan la inequidad (Hernández \& Camarena, 2005). El techo de cristal hace referencia a las barreras no explícitas que impiden a las mujeres acceder a ciertos cargos directivos y constituyen un obstáculo que les impide ejercer roles de liderazgo, lo cual, al no estar regulado ni controlado, permanece invisible a los ojos de la sociedad (Bucheli \& Sanroman, 2004). Un ejemplo de la existencia del techo de cristal es que la promoción hacia altos cargos directivos resulta ser más selectiva debido al grado de responsabilidad, autoridad y riesgo para la compañía, todas ellas características que al no corresponder con el estereotipo femenino, pueden limitar el acceso de las mujeres (Yap \& Konrad, 2009).

Otro aspecto en el que es posible observar una diferencia importante a nivel de liderazgo empresarial, es el emprendimiento. Guzmán \& Rodríguez (2008) señalan que aunque la distancia entre hombres y mujeres que impulsan la creación de nuevas empresas se ha reducido notablemente, siguen siendo los hombres quienes continúan 
liderando con mayor frecuencia estas iniciativas. Dichos autores afirman que el progreso de las mujeres en este aspecto obedeció en gran medida al acceso a la educación, a su participación cada vez más amplia en el mercado laboral (no solo en cargos directivos sino en cargos inferiores o de gerencia media) y al auge del sector de los servicios en el que las mujeres tradicionalmente se han desempeñado.

En la actualidad se puede evidenciar el resultado de importantes procesos de cambios sociales, culturales y demográficos sobre el rol de las mujeres. Es notable el creciente ingreso del género femenino en el mercado laboral, fenómeno que está sucediendo a nivel mundial y Colombia no es la excepción, pues el $40 \%$ de la fuerza laboral del país está constituida por mujeres. Así mismo, la composición familiar ha ido cambiando, se ha incrementado la jefatura femenina, hay mayor acceso a la educación y una marcada reducción de la fertilidad, entre otros (Arriagada, 1997; Duryea, Cox \& Ureta, 2006). Estos últimos dos aspectos guardan una estrecha relación con el ingreso de las mujeres al mundo laboral y, seguramente, se verá reflejado en su ascenso en la jerarquía empresarial. De acuerdo con varios estudios, a mayor nivel de escolaridad y menores tasas de fecundidad la participación de la mujer en la fuerza laboral será más elevada (Duryea et ál., 2006; Abramo \& Valenzuela, 2006), situación que ya se está observando en Colombia (Figura 1).

INDICADORES DE FECUNDIDAD EN COLOMBIA

1985-2020

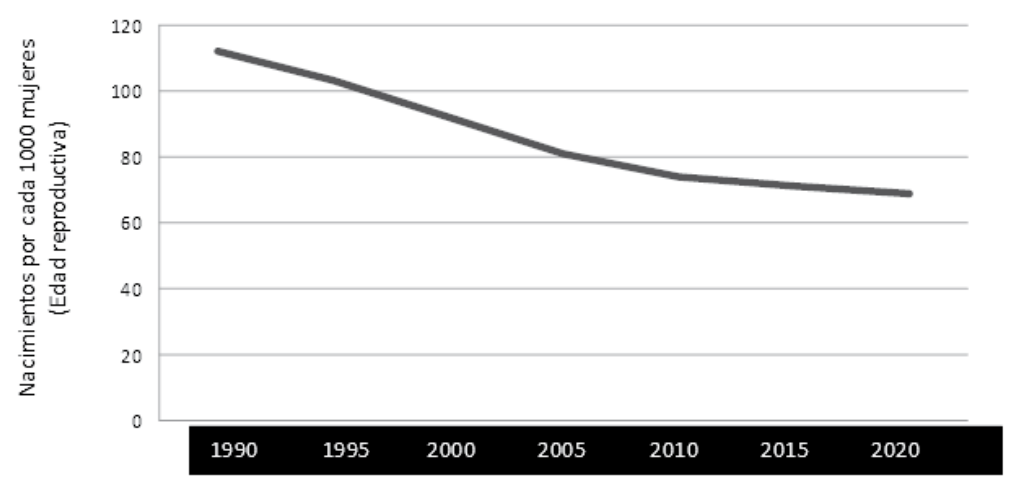

Figura 1. Datos tomados de DANE, conciliación censual 1985-2005 y proyecciones de población 2005-2020, citados por el Ministerio de la Protección Social (s.f).

En Colombia, este inminente ascenso de las mujeres ha sido también producto de los esfuerzos de varias empresas por corregir los sesgos contra la paridad en altos cargos de liderazgo y de la llamada ley de cuotas del año 2000, la cual reglamenta la participación femenina en por lo menos en un $30 \%$ de los cargos de alta dirección del Estado (Carter \& Silva, 2010).
Como resultado de todos estos procesos, se puede observar también un cambio importante $y$ progresivo en los ámbitos en los cuales la mujer tradicionalmente se desempeñaba, destacándose en trabajos considerados casi exclusivamente masculinos y en los que ha incursionado con mucha fuerza, tal es el caso del sector salud, las Tecnologías de la Información y la Comunicación 
(TIC), las ciencias de la computación, leyes e ingeniería (OIT, 2004). En Colombia, particularmente, son notables sus avances en el sector financiero (Tenjo, 2000).

Cabría preguntarse entonces, ¿cuáles pueden ser las implicaciones de este inminente (aunque no tan rápido como se esperaría) ascenso de las mujeres en cargos de liderazgo?, y ¿cuál será su papel frente a las exigencias que requieren los desafíos empresariales que demanda un mundo globalizado y competitivo como el actual? Responder a estas preguntas implica una comprensión de las diferencias inherentes que existen entre hombres $y$ mujeres y que pueden constituir una riqueza para la organización. No cabe la menor duda de que la diversidad es un activo para las organizaciones y el liderazgo entre unos y otros puede fortalecer a las empresas y permitirles adaptarse mejor a los vertiginosos, impredecibles y rápidos cambios del mundo moderno. Desde esta perspectiva, se resalta el papel de la diferencia con equidad, que es diferente de mismidad. No se trata de copiar roles masculinos sino de liderar desde las características propias relativas al sexo como seres biológicos y al género, como construcción social que sustenta dichos roles.

\section{Roles de liderazgo y género}

Las condiciones socioeconómicas y políticas en las que se encuentran inmersas las empresas hoy se caracterizan por un alto grado de incertidumbre, escaso nivel de predicción, entornos altamente complejos, globalización, economías emergentes e interdependientes, mayor competencia mundial, tendencias más globales que locales y cambios demográficos y tecnológicos (Lipman-Blumen, 2000). Estas circunstancias imponen a las organizaciones y a los líderes grandes e importantes retos.

Asumir la diversidad implica reconocer que existen diferencias de carácter psicológico, biológico, social y cultural que influyen ineludiblemente en el comportamiento de hombres y mujeres. Es importante aclarar que el sexo es un concepto biológico, mientras que el género es una concepción más amplia, que en palabras de Gálvez (2001) define los roles y los valores asociados a cada uno, teniendo en cuenta factores históricos y sociales. Es este concepto el que sustenta los estereotipos, creencias culturalmente compartidas, que pueden limitar el liderazgo en las mujeres.

La concepción acerca del género se basa en la idea de que la realidad, tanto de hombres como de mujeres, es asumida de forma diferente dando lugar a categorías que demarcan diferencias dentro de una sociedad. Estas categorías, a su vez, se componen de percepciones individuales respecto al rol de hombres y mujeres (Constantinidis, 2010). Este proceso llamado categorización social indica que las personas adoptan ciertos filtros y sesgos acerca de determinados grupos de individuos que pertenecen a una categoría y condicionan la manera como las personas se perciben unas a otras. Es así como se construye un criterio sociocultural que permanece en el imaginario colectivo de la sociedad y genera una representación compartida acerca de ciertas características propias tanto del género masculino como del femenino (Uribe, Manzur, Hidalgo \& Fernández, 2008).

Estas creencias comienzan a verse reforzadas en comportamientos característicos que desde la niñez se imprimen y comienzan a modular la personalidad, a través de un proceso llamado aprendizaje observacional (Schiffman \& Kanuk, 2005), en el cual los niños comienzan a imitar las características atribuidas a cada género. Basta simplemente con observar el comportamiento infantil y cómo difiere entre niños y niñas, tanto por la influencia del entorno social que los rodea como por sus características biológicas que de hecho distinguen y modulan su comportamiento. Villanueva \& Nancy (2005) realizaron un estudio en el que se evidenció que los niños demuestran habilidad para conformar grupos y organizar juegos de competencia y fuerza física, desarrollan más rápido el razonamiento aritmético y espacial, y tienen mayor dinamismo corporal. Por otro lado, Dunker (2002) observó que las niñas muestran una actitud más formal, les preocupa su apariencia física, sus comportamientos se orientan hacia las conversaciones, son más tranquilas, se fijan en los detalles, responden a varios estímulos a la vez y 
desarrollan más rápidamente un lenguaje fluido. Así mismo, demuestran habitualmente tener más éxito en labores estéticas y verbales, presentan más preocupaciones sociales, suelen integrarse mejor, ayudan, comparten y cooperan. Los niños son más hábiles en tareas que implican aptitudes espaciales, razonamiento lógico y definición de palabras abstractas y suelen ser también más agresivos, incluso, afirma este autor, que en las mujeres predomina la inteligencia emocional y en los hombres la inteligencia racional.

Lo anterior podría explicar el porqué hay una mayor proporción de mujeres en roles de apoyo. Clancy (2007) sostiene que a diferencia de los hombres, las mujeres suelen ser más empáticas y se enfocan principalmente hacia el consenso y la afiliación, las ubica en posiciones en las que si bien no asumen un poder significativo, logran satisfacer sus intereses personales, como parte de una elección propia y no debido a condiciones de inequidad.

Dadas las diferencias descritas anteriormente, el estudio sobre el liderazgo femenino podría enfocarse hacia el análisis de cómo las características propias (no exclusivas) de cada género ejercen algún tipo de influencia sobre sus prácticas directivas. Desde esta perspectiva, Eagly \& Carli (2007) afirman que los roles masculinos y femeninos de liderazgo se diferencian en dos aspectos básicamente, uno es el que ellos llaman comunal y el otro el de poder. De acuerdo con estos autores, las mujeres estarían más orientadas hacia las relaciones interpersonales, serían más afectivas, emocionales y sensibles, mientras que los hombres acudirían más al control, a la agresividad, a la dominación, a la ambición y al individualismo. Echavarri, Godoy \& Olaz (2007) encontraron también diferencias de género respecto a la forma de resolver problemas complejos: mientras que los varones utilizan más el razonamiento (espacial y abstracto), las mujeres recurren más al uso de estrategias verbales.

En concordancia con lo anterior, Rosener (1990) afirma que características generalmente asociadas a las mujeres, como el carisma y las habilidades sociales, son las que marcan la diferencia y conforman un nuevo modelo que la misma autora denomina liderazgo interactivo, tomando en consideración algunos rasgos típicos de su condición como la conciliación y el empoderamiento de sus equipos con base en interacciones positivas entre los involucrados. Por otro lado, Arteaga \& Ramón (2009) enmarcan las características de lo que ellos llaman el liderazgo resonante, el cual se concentra principalmente en la orientación de las emociones de un equipo hacia el logro de los objetivos, propiciando el trabajo colectivo, la cohesión social y la integración, incluso en situaciones adversas.

Dunker (2002) por su parte, ha encontrado que los hombres y las mujeres usan estilos diferentes de liderazgo: mientras las mujeres tienden a ser más democráticas, participativas, menos autocráticas que los hombres, valoran la filiación y la adhesión; por el contrario, los hombres tienden a emplear un estilo más directivo, centrado en el mando y el control, el poder y la competencia, ya que confían en la autoridad formal de su posición como base para ejercer su influencia.

No obstante, atribuir tales diferencias en razón de las características asociadas al sexo y al género ha recibido importantes críticas. Hossain \& Barry (2010) señalan que no es como identificar genéticamente el color de los ojos o la cultura por el acento, esta distinción es casi imposible cuando se analizan comportamientos asociados con el liderazgo, los cuales no pueden universalizarse pues este es situacional y contextual. Lo que está claro es que la percepción social del género crea diferentes características a nivel físico, psicológico, de pensamiento, cultural y de aprendizaje, etc., que hacen que este concepto se perciba de diferentes maneras en todas las esferas $y$, definitivamente, las organizaciones no son la excepción (Al-Jenaibi, 2010).

Si se asume entonces que hombres y mujeres son distintos, el liderazgo, definido como una serie de comportamientos que implican la comunicación, la forma en que se administran los conflictos, la toma de decisiones y demás, traerá consigo ciertas particularidades en razón de tales diferencias. No se pretende decir que alcanzar resultados 
exitosos en posiciones de liderazgo dependa de si se es hombre o mujer sino que la forma en que se ejerce el liderazgo puede ser diferente, de acuerdo con las características asociadas tradicionalmente a cada género, y que algunas de ellas, se pueden acoplar mejor a ciertas condiciones del entorno (Rosener, 1990).

Debido a lo anterior, Gálvez (2001) plantea que la discusión en torno al género debe centrarse en el reconocimiento de tales diferencias e incluso exaltarlas en un contexto de equidad, es decir, en un marco de oportunidades asequibles para todos. De esta manera, el que las organizaciones posean líderes hombres y mujeres, constituirá una fuente de riqueza en cuanto la diversidad en sí misma lo es.

\section{Factores asociados con la inequidad}

La globalización, el desarrollo tecnológico y la situación económica han ocupado lugares prioritarios en el contexto mundial desde la última década del siglo $X X$, ubicando la discusión en torno a la equidad de género en un plano menos visible (Yannoulas, 2005). Sin embargo, desde los años setenta se han desarrollado varias iniciativas para incidir en las políticas públicas en favor de la equidad, a través de eventos como la Conferencia Mundial de la Mujer que tuvo lugar en México (1975), Nairobi (1985) y Beijing (1994), solo por citar algunos cuantos, a través de los cuales se logró introducir el enfoque del género en todas las políticas y acciones gubernamentales tendientes a fortalecer la inclusión de la mujer (Longo, 2009).

Sin embargo, al parecer el problema fundamental para no lograr condiciones de equidad ha sido principalmente de carácter socio-cultural, esto es, el afianzamiento de los roles tradicionales, sostenidos incluso por muchas mujeres, quienes consideran que los hombres deben asumir su rol de proveedor mientras que la mujer debe asumir la responsabilidad del hogar, desvirtuándose la capacidad femenina en el campo económico, político y educativo (Gálvez, 2001). Esta explica- ción es compartida por otros autores como Clancy (2007), quien señala que los prejuicios en torno a los roles femeninos no son originados solamente por los hombres sino que son las mujeres las que apoyan el estereotipo de inferioridad femenina en el campo laboral, situación que conlleva a que persista la discriminación en términos de remuneración, segregación vertical (concentración del empleo femenino en la base de la jerarquía) y segregación horizontal (concentración del empleo femenino en aquellos sectores afines con su rol) (Maruani, 1993). Este último aspecto incide en el nivel de desempleo en las mujeres, el cual aunque ha mejorado notablemente, aún sigue siendo alto dado que muchos trabajos no están disponibles para las mujeres por los roles socialmente asignados a su género (Aguirre, 2001). De acuerdo con el DANE (2011), en Colombia la tasa de desempleo femenina fue significativamente mayor que la masculina $(16,8 \%$, y $9,2 \%$, respectivamente).

La segregación también se mantiene debido a las políticas de asignación y jerarquización de funciones organizacionales, las cuales frecuentemente utilizan como indicadores de evaluación el nivel de compromiso hacia la empresa, que en el caso de las mujeres está sujeto principalmente a sus responsabilidades en el hogar. Este hecho condiciona su posición y en ocasiones la pone en conflicto con otras dimensiones de su proyecto de vida personal (Hernández \& Camarena, 2005), y constituye una barrera importante al considerar la maternidad como una desventaja para la empresa en términos de costos, limitando así la promoción de las mujeres en cargos directivos (López, Martin \& Romero, 2009).

De igual forma, algunos procesos de reclutamiento y de selección como parte de los procesos de Gestión Humana pueden contribuir a mantener la inequidad, tal como lo señalan Fielden \& Davidson (1997), la carencia de procedimientos formalizados y estandarizados para ocupar las vacantes de altos cargos directivos fomenta la reproducción de la inequidad; muchas empresas, especialmente las pequeñas y las medianas, utilizan las "recomendaciones" como fuente de selección a falta de un proceso formal que lo sustente, y dado que hay mayoría de hombres en estos cargos, 
estos suelen recomendar hombres para ocupar las vacantes, perpetuándose así esta condición. Así mismo, la baja participación de la mujer en cargos directivos se explica por la poca experiencia en dichas posiciones, debido justamente a la falta de oportunidad para acceder a ellas lo cual va configurando un círculo vicioso que limita su ascenso.

Lo anterior genera un reto importante para la gestión del talento humano en las organizaciones, que implica replantear la definición de roles y responsabilidades en el campo laboral, fundamentado principalmente en un modelo masculino que deja de lado la naturaleza misma de la condición femenina (Iranzo \& Richter, 2002) y contribuye al fortalecimiento de los estereotipos de género asignando un rol subordinado a la mujer (Longo, 2009). Al respecto, Abramo (2004) señala que tanto desde la concepción social como desde la misma cultura empresarial la mujer continua siendo una fuerza laboral secundaria frente al hombre, a pesar de los avances que se han alcanzado en este aspecto.

No cabe duda de que el embarazo, el matrimonio, la familia y el cuidado de los hijos entran a formar parte de las razones por las cuales existen diferencias en el rol de hombres y mujeres, y que en cierta medida pueden también limitar su ascenso en la pirámide organizacional (Escandón \& Arias, 2011); no obstante, como plantea Clancy (2001), ejercer el rol no debe tomarse como una limitante y no debe ser visto siempre como resultado de la discriminación sino como una elección personal que las mujeres deben tener la libertad de tomar.

La limitante fundamental para ascender a altos cargos directivos va más allá de estas diferencias, depende más de los estereotipos comunes que manifiestan la idea de que el éxito gerencial está asociado a las características de los hombres y no a las de las mujeres. Al respecto, GarcíaRetamero \& López-Zafra (2006) encontraron en su estudio que la atribución de éxito o fracaso en el ejercicio del liderazgo depende en gran medida de la percepción de incongruencia entre el rol de género y el rol de líder, es lo que Eagly \& Karau (2002) han llamado la Teoría de Congruencia de
Rol. García-Retamero \& López-Zafra (2006) afirman que las creencias y la percepción de incongruencia ejercen un efecto importante y podrían ser la base del prejuicio. De acuerdo con estos autores, en la medida en que una mujer ocupa un puesto de trabajo evaluado como incongruente con su rol, la percepción de incongruencia se acentúa, y se atenúa cuando este es evaluado como apropiado para su rol.

Hay que tener en cuenta que en muchas sociedades, la definición de un buen líder tiene marcados rasgos masculinos, que van en contravía con las características asociadas a las mujeres, lo cual explicaría por qué, en ocasiones, algunas mujeres que alcanzan altos cargos directivos pueden comportarse de manera más ruda, controladora y menos sensible que lo que harían sus contrapartes masculinos.

Así, los prejuicios que relacionan rasgos y conductas masculinas con el desempeño de roles de liderazgo constituyen uno de los mayores obstáculos que deben enfrentar las mujeres a la hora de acceder a niveles jerárquicos más altos; es más, aquellas que ocupan altos cargos de liderazgo se enfrentan ante una exigencia mayor que la esperada si fuera hombre, considerando que ellas deben probar tanto su capacidad como su potencial para ocupar estos cargos (Godoy \& Mladinic, 2009).

Finalmente, Delfino (2004) señala la necesidad de una reingeniería de las estructuras empresariales tradicionalmente lideradas por el género masculino, dando paso a un modelo de gestión que rompa este esquema y permita la expresión de las habilidades femeninas como oportunidades de flexibilización de estos sistemas. Godoy \& Mladinic (2009) consideran la conveniencia de incluir dichas habilidades en el desarrollo de las organizaciones, dada la necesidad de flexibilizar su estructura y contar con un estilo de liderazgo participativo y transformacional, que según la psicología social se puede asociar más con el perfil femenino. De acuerdo con esta posición, Newman (1995) señala que una cultura transformacional podrá brindar mayores posibilidades de desarrollo y ascenso 
para las mujeres en cuanto el liderazgo desde que esta perspectiva esté centrada en las personas.

\section{Comentarios finales}

La anterior revisión respecto al liderazgo femenino deja más interrogantes que certezas en cuanto al futuro. Aunque las mujeres han alcanzado un importante posicionamiento a nivel laboral, en comparación con décadas anteriores, aún existen importantes brechas que les limita la posibilidad de alcanzar cargos de alta dirección.

Las barreras para alcanzar tales posiciones van más allá de la formación, pues hoy en día hay paridad a nivel educativo, incluso las mujeres en diferentes ámbitos han superado al varón, el problema está más centrado en los estereotipos vinculados al género, los cuales están en clara disonancia con el rol de liderazgo, que suele estar compuesto por características masculinas. Con base en lo anterior, podría decirse que una de las barreras más importantes que se debe superar es la concepción de que la mujer no sería buena líder dado que no va con su naturaleza.

El reto para las organizaciones modernas, y para la mujer dentro de ellas, es reconocer y potenciar la diferencia como una fuente de riqueza. Los líderes son individuos y sus prácticas de liderazgo dependen, entre otras cosas, de las características personales y el género hace parte de ellas. La propuesta es, entonces, propender por la equidad no por la igualdad y reconocer que el contar con altos directivos, hombres y mujeres, esta diversidad podrá ser vista como una ventaja competitiva a nivel organizacional.

Por otra parte, es inminente el ascenso de las mujeres en la escala empresarial, los datos así lo señalan: disminución en las tasas de natalidad, mayor nivel de estudio, incremento de la jefatura femenina, entre otros. Lo anterior constituye un reto para la gestión del talento humano y para la alta gerencia: evitar la discriminación, las diferencias salariales, la inequidad en la participación directiva que no permite a algunas mujeres tener experiencia en ellas y perpetúa la dispari- dad. Cabe también aclarar, que el ocupar un cargo en la alta dirección puede ser visto también como una opción para la mujer, sobre la cual ella debe decidir, pues muchas mujeres no ascienden, no por discriminación, ni por inequidad sino por tener intereses diversos y el laboral puede no ser el primordial.

Valdría la pena reconocer la importancia que las mujeres logren desarrollar un liderazgo acorde con sus propias características que en sí mismas ya son diversas, el imitar el patrón masculino puede ser adverso en este sentido, pues las mujeres pueden llegar a ser más rudas que los hombres, lo cual desvirtuará su propia capacidad de liderazgo.

Es importante señalar que la inequidad en los cargos de alta dirección sigue siendo un fenómeno sin dilucidar, del que se tienen varios indicios, y en el que el factor socio-cultural parece ser el más relevante. No obstante, aún hay muchos interrogantes que deben ser develados con el fin de intervenir favorablemente con acciones que promuevan la equidad a nivel laboral. Lo anterior requiere el desarrollo de estudios culturales de carácter aplicado, que permitan comprender los prejuicios que sustentan la marcada distinción de roles y las creencias respecto a las actividades que pueden ser ejercidas por hombres y mujeres en una sociedad determinada. Cabe recordar que el liderazgo es un concepto histórico, social y cultural, es decir, un líder es considerado como tal, según las percepciones y las creencias que una sociedad tenga respecto a este.

Finalmente, reconocer las diferencias entre hombres y mujeres, y a través de ellas lograr la equidad es un reto importante y necesario en el mundo moderno, en el que se reconoce ampliamente la importancia de la diversidad. El logro de este propósito requiere continuar haciendo estudios transdisciplinarios que permitan comprender las diferencias biológicas, psicológicas y sociales entre hombres y mujeres tanto a nivel general, como organizacional, con mujeres que ejerzan roles de liderazgo. En este caso, la comparación entre culturas ayudaría también a comprender mejor el fenómeno, dado su impacto sobre el concepto de liderazgo. 


\section{Referencias}

Abramo, L. (2004 ). ¿Inserción laboral de las mujeres en América Latina: una fuerza de trabajo secundaria? Revista Estudios Feministas, 2, 224-235.

Abramo, L. \& Valenzuela, M. E. (2006). Inserción laboral y brechas de equidad en América Latina. En L. Abramo (Ed.). Trabajo decente y equidad de género en América Latina (pp. 29-62). Santiago de Chile, Chile: Oficina Internacional del Trabajo.

Aguirre, R. (2001). Trabajo y género. Caminos por recorrer. En R. Aguirre \& K. Batthyány (Eds.). Trabajo, género y ciudadanía en los países del Cono Sur (pp. 165-180). Montevideo, Uruguay: Oficina Internacional del Trabajo.

Al-Jenaibi, B. (2010). Differences between gender treatments in the Work Force. Cross-Cultural Communication, 6(2), 63-74.

Arriagada, I. (1997, agosto). Realidades y mitos del trabajo femenino urbano en América Latina. Santiago de Chile: Unidad Mujer y Desarrollo, Comisión Económica para América Latina y el Caribe. Naciones Unidas.

Arteaga, A. \& Ramón, S. (2009). Liderazgo resonante según género. Multiciencias, 9, 289-295.

Bucheli, M. \& Sanroman, G. (2005). Salarios Femeninos en el Uruguay ¿Existe un Techo de Cristal? Revista de Economía del Banco Central del Uruguay, 12(2), 63-88.

Carter, N. M. \& Silva, C. (2010). Mentoring: Necessary but insufficient for advancement. New York: Catalyst.

Clancy, S. (2007). ¿Por qué no hay más mujeres en la cima de la escala corporativa: debido a estereotipos, a diferencias biológicas o a escogencias personales? Revista Latinoamericana de Administración, 38, 1-8.
Comisión Económica para América Latina y el Caribe (CEPAL) (2010). Que Estado para que igualdad? En XI Conferencia Regional sobre la Mujer de América Latina y el Caribe, 13-16 de julio de 2010. Brasilia. Recuperado el 25 de agosto de 2011, de: http://www.eclac.org/ publicaciones/xml/6/40116/Que_Estado_ para_que_igualdad.pdf

Constantinidis, C. (2010). Représentations sur le genre et réseauxd'affaireschez les femmes entrepreneures. Revue Française de Gestion, 36, 127-143.

Delfino, A. (2004). Flexibilidad y rigidez: La permanencia de las representaciones sobre lo femenino en un contexto de transformaciones productivas. Ámbitos, 11-12, 263-279.

Departamento Administrativo Nacional de Estadística (DANE). (2011, 13 de mayo). Mercado laboral por sexo "Gran encuesta integrada de hogares trimestre enero-marzo 2011". Recuperado el 20 de septiembre de 2011 de: http://www.dane.gov.co/files/investigaciones/boletines/ech/ech/bol_sexo_abr_jun11. pdf

Dunker, L. (2002). Iguales y diferentes. Un estudio sobre género, matrimonio y familia. Argentina: El Cid.

Duryea, S., Cox, A. \& Ureta, M. (2006). La mujer en el mercado de trabajo: los extraordinarios años noventa en América Latina. En C. Piras (Ed.). Mujeres y trabajo en América Latina. Desafíos para las políticas laborales (pp. 2960).Washington, DC: BID.

Eagly, A. \& Carli, L. (2007, septiembre). Women and the labyrinth of leadership. Harvard businessreview, 1-8. Recuperado de: http://citt. hccfl.edu/Newsletters/NewsletterID1.pdf

Eagly, A. H. \& Karau, S. J. (2002). Role congruity theory of prejudice toward female leaders. Psychological Review, 109, 573-598. 
Echavarri, M. Godoy, J. C. \& Olaz, F. (2007). Diferencias de género en habilidades cognitivas y rendimiento académico en estudiantes universitarios. Universitas Psychologica, 6(2), 319-329.

Escandón, D. \& Arias, A. (2011). Factores que componen la competitividad de las empresas creadas por mujeres y las relaciones entre ellos. Cuadernos de Administración, 24(42), 165-181.

Fernández, M. P. (2006). Determinantes del diferencial salarial por género en Colombia, 19972003. Desarrollo y sociedad, 58, 165-208.

Fielden, S. \& Davidson, M. (1997). Equal Opportunities in Recruitment: The Job Search Experiences of Unemployed Female and Male Managers. Equal Opportunities International, 16(6-7), 50-59.

Gálvez, T. (2001). Aspectos económicos de la equidad de género. Unidad mujer y desarrollo, 35. Santiago de Chile: CEPAL. Recuperado el 12 de febrero de 2011 de: http://www.eclac. cl/publicaciones/xml/4/7374/lcl1561e.pdf

García-Retamero, R. \& López-Zafra, E. (2006). Congruencia de rol de género y liderazgo: el papel de las atribuciones causales sobre el éxito y el fracaso. Revista Latinoamericana de Psicología, 38(2), 245-257.

Godoy, L. \& Mladinic, A. (2009). Estereotipos y Roles de Género en la Evaluación Laboral y Personal de Hombres y Mujeres en Cargos de Dirección. Psykhe, 18(2), 51-64.

Guzmán, J. \& Rodríguez, M. J. (2008). Comportamiento de las mujeres empresarias: una visión global. Revista de Economía Mundial, 18, 384-385.

Henrekson, M. \& Stenkula, M. (2009). Why are there so few female top executives in Egalitarian Welfare States. The Independent Review, 14(2), 239-270.
Hernández, C. \& Camarena, M. E. (2005). Inequidad de género en las organizaciones. Una visión de Latinoamérica. Actualidad Contable Faces, 8, 20-29.

Hossain, F. \& Barry, P. (2010). Leadership through a gender lens: How Cultural Environments and Theoretical Perspectives Interact with Gender. International Journal of Public Administration, 33, 81-87.

Iranzo, C. \& Richter, J. (2002). El espacio femenino en el mundo del trabajo formal. Revista Venezolana de Gerencia, 7, 509-535.

Lipman-Blumen, J. (2000). Connective leadership: managing in a changing world. USA: Oxford University Press.

Longo, M. E. (2009). Género y trayectorias laborales. Un análisis del entramado permanente de exclusiones en el trabajo. Trayectorias, $11,118-141$.

López, M., Martin, A. F. \& Romero, P. (2009). Key factors in the Access of women to managerial posts. Journal of General Management, 34(4), 39-50.

Maruani, M. (1993). La cualificación, una construcción social sexuada. Economía y sociología de trabajo, 21, 41-50.

Maxfield, S. (2007). Women on the Verge of Corporate Power in Latin America II. Boston, MA: Center for Gender in Organizations, Simmons College.

Ministerio de Protección Social, Dirección General de Promoción del Trabajo. (s.f). Población y principales indicadores demográficos de Colombia. Recuperado el 7 de marzo de 2011 de: http://www.griunad.com/omt/ omt_meta/docs/MODULOS/DEMOGRAFIA/ Poblacion\%20y\%20principales\%20indicadores\%20demograficos\%20de\%20Colombia.pdf

Newman, J. (1995). Gender and cultural change. En C. Itzin \& J. Newman (Eds.). Gender, 
Culture and Organizational Change (pp. 1129). New York: Routledge.

Organización Internacional del Trabajo (OIT). (2004). Panorama Laboral 2004. América Latina y el Caribe. Recuperado el 24 de marzo de 2011 de: http://www.oit.org.pe/portal/ documentos/panorama_laboral_2004.pdf

Osca, A. \& López-Sáez, M. (1994). Desarrollo de carrera y género: factores que influyen en las diferencias entre hombres y mujeres. Revista de Psicología del Trabajo y de las Organizaciones, 10(28), 73-86.

Rodríguez de Lecea, T. (2008). Equidad de género. Tiempo de Paz, 89, 89-90.

Rosener, J. (1990, noviembre). Ways women lead. Harvard business review, 119-125.

Sánchez, R. (1991). Vigencia de las ideas de la revolución francesa en la América Latina del siglo XX. Historia crítica, 5, 99-106.

Schiffman, K. \& Kanuk, L. (2005). Comportamiento del consumidor. México: Pearson Prentice Hall.
Tenjo, J. (2000). Evolución del empleo y los salarios de hombres y mujeres en el sector financiero. Estudios sobre Economía y Género. Bogotá: Proequidad / GTZ.

Uribe, R., Manzur, E., Hidalgo, P. \& Fernández R. (2008). Estereotipos de género en la publicidad: un análisis de contenido de las revistas chilenas. Revista Latinoamericana de Administración, 41,1-18.

Villanueva, V., \& Nancy, B. (2005). Socialización y comportamiento infantil según el género. Red Mitológicas, 35-41.

Yannoulas, S. C. (2005). Perspectivas de género y políticas de formación e inserción laboral en América Latina. Buenos Aires: redEtis.

Yap, M. \& Konrad, A. M. (2009). Gender and Racial Differentials in Promotions: Is There a Sticky Floor, a Mid-Level Bottleneck, or a Glass Ceiling?. Relations industrielles / Industrial Relations, 64(4), 593-619. 